Letter to the Editor

\title{
Shedding light on the restart
}

\section{A R T I C L E I N F O}

\section{Keywords:}

Antimicrobic phototherapy

Germicidal UV

UV safety

UVC

COVID-19

According to Shaman and Kohn [1] there are four ways for viral infection transmission: (i) through direct physical contact with an infected individual; (ii) via intermediate objects; (iii) via droplets expelled from infected individuals (e.g., by sneezing or coughing) that deposit on nasal or oral mucosa of a susceptible individual; (iv) via expelled particles $<2.5 \mu \mathrm{m}$ in radius (droplet nuclei) that remain suspended in air as aerosols for extended periods of time. On the same subject, the World Health Organization has recently released a commentary [2].

Apart from the first point, we believe that the medical physicist can (or better: should) actively participate in the debate and the consequent actions to be taken in order to cope at best with the SARS-CoV-2 pandemic and its consequences at a sanitary, social and economic level. Of course, we consider it paramount to frame the contribution of medical physics in an inter-disciplinary context, where synergy and cooperation between different disciplines is the key to achieve a successful outcome.

Generally speaking, ionizing radiations are certainly known to be associated with antimicrobial effects: gamma rays, X-rays and electron beams can be used for disinfection or sterilization purposes even if the necessary doses are often of the order of kGy or more [3]. It being understood their clear interest for the medical physicist, these radiation types and the relative sources are in our opinion much more difficult to insert in an everyday human-populated context rather than UV sources, corresponding to the elective radiation type found in literature studies on antimicrobial solutions.

In this context, our reflection starts from basic considerations taken from the relevant literature, including in particular recent results about viral infection transmission and means to contain or eradicate it by UV-visible radiation as an effective antimicrobial agent [4,5]. Before focusing on this point, we would like to recall two aspects concerning viral infections that, in our opinion, can drive the medical physicist towards specific conclusions on the best possible countermeasures with physical means.

From the data available in the literature $[6,7]$ in a single cough we emit about 5000 particles whose dimensions vary from: (i) $>80 \mu \mathrm{m}$ (6\% particles) accounting for $99 \%$ of the total particle volume, readily falling to the ground; (ii) $40-80 \mu \mathrm{m}(5 \%)$ aggregating into $>100 \mu \mathrm{m}$ particles in case of high relative humidity $(\mathrm{RH}=90 \%)$ while evaporating into $<40 \mu \mathrm{m}$ particles otherwise; (iii) $<40 \mu \mathrm{m}(89 \%)$ remaining long in air (up to few hours) due to their negligible weight compared to other driving forces $[8,9]$. Likewise, in a single sneeze most particles are in the $<40 \mu \mathrm{m}$ range (96\%), while only $2 \%$ over $80 \mu \mathrm{m}$. This indicates the importance of addressing the problem of viral load transported by the smallest particles in air, especially in closed spaces such as hospitals, offices, shops and public transport.

The second aspect is the possible influence of environmental conditions on infection diffusion.

Such conditions can be represented by physical variables (e.g. temperature, relative air humidity, solar irradiance) whose influence can be largely studied by physical modelling, like in the case of droplet dimensions, evaporation and permanence in the air and the factors controlling them.

In many respiratory infection cases a dependence of infectivity on seasonal variables has been observed [1,10-12], some authors suggesting a correlation with seasonal variation in vitamin D serum concentration [13]. In our view, these data are consistent with the sure increase in ambient sunlight irradiance and in particular of its UV-violet components [14].

Starting from these observations, we would like to emphasize that UV-violet light in general may have a direct antimicrobial effect depending upon wavelength and dose, and that sunlight irradiance in the UV region is certainly a well-known seasonal phenomenon especially for temperate latitudes [14].

In the environment UV solar radiation is the main natural virucide [15] even if providing UVA and UVB but not UVC, at ground level. UV radiation kills viruses by chemically altering their material. The most effective wavelengths for inactivation are those that are most absorbed by DNA and RNA, precisely corresponding to the UVC range (100-280 nm). Nucleic acids are also damaged by UVB (280-315) and UVA (315-380 nm) but with lower efficiency [15-17].

Interestingly enough, the atmosphere cuts out practically all UVC and part of UVB solar radiation, which are known to be the most effective antimicrobial wavelengths $[4,16]$ excluding the use of external photosensitizing molecules [18], making studies on the sterilizing efficacy and applicability of these radiations even more intriguing. Even if it is obvious that the chemo-physical and biological principles of UVphotokilling efficacy stand independently on the physical location of microbes (i.e. in the environment or in our body), still the most easily 
achievable and accessible applications of UV as an antimicrobial agent are probably in environmental disinfection. In this field, the use of UV in the presence of people has always been curbed by the pathogenic effects that exposure to these wavelengths could have on the skin and cornea $[19,20]$. This is certainly a research area deserving further studies, especially if UVC should be increasingly used for viral disinfection: due to UVC limited penetration in the skin, erythemal and mutagenic effects on deep proliferating cells are not known. Still, possible effects on the skin and the cornea would need to be studied (e.g. long-exposure ones), especially if new and more powerful sources should reach the market.

All these considerations indicate that we as medical physicists should probably focus our attention on UV radiation now more than ever, and namely on UV-sources, UV-dosimetry and UV-induced biological effects (intended both as antimicrobial effects and in terms of radioprotection), to better understand and design irradiation strategies and effectively deal with prevention and protection issues.

From the point of view of UV sources, in the last years new solutions have come to the market, alongside "traditional" ones such as Hg-vapor lamps or excimer lasers, like for example new excimer lamps with increasing efficacy (up to $\sim 40 \%$ ) also in the far UVC range which have been recently considered for disinfection purposes $[4,5]$. In general, the quantum efficiency of LED emission drastically depends on the wavelength. LEDs with InGaN technology $(365-400 \mathrm{~nm})$ show similar performances to blue LEDs, sharing an analogous technology (see Nobel Prize for Physics, 2014). AlGaN-based devices, with emission wavelength below $365 \mathrm{~nm}$, have single-digit efficiencies, although improvements have been recently published for UV LEDs near $275 \mathrm{~nm}$ [21].

Talking about lamps, they could be preferable in the short time span due to their higher radiant power, great emission solid angle and the presence of ready solutions for room disinfection (e.g. in hospitals) especially for the air and as a help for surfaces. Nevertheless, they tend to be bulky and costly; besides, irradiation geometry and timing are hardly controllable. For these reasons they are mainly used for room disinfection at night (where no occupants are present, a limiting factor itself) and/or they need the intervention of trained personnel for radiation release and control, unless they are used e.g. in between visits in medical clinics.

Therefore, in a longer perspective LED sources could be far more flexible, provided they will reach a "satisfactory" emission in terms of radiant power and peak wavelength availability. In fact, LED emission can be easily controlled in terms of both time-modulated power and beam geometry shaping, due to their intrinsically small dimensions ("punctiform sources") and functioning principle. This will facilitate their integration in closed environments where occupants never leave the room (e.g. intensive care rooms, corridors and, by extension, offices, shops and transport means) avoiding the need for a specific "disinfection time", being LED emission integrated in the normal activities with very low impact on their schedule and progress. Some solutions are already implemented e.g. in terms of UV lamps generating "light strips" in the highest volume section of a room, relying on normal air movement to maximise their disinfecting capacity towards bio-aerosol. Even in this simple case, a careful modelling of light propagation (for disinfection purposes) and diffuse reflection by walls (for radioprotection issues) is needed by dedicated staff. This is even more necessary and interesting thinking of a possible and synergic integration of UV irradiation with artificial intelligence solutions, where LED or laser beams could be interrupted while the presence of an individual is detected in the surroundings of the beam route.

Speaking about UV dosimetry, we can frame it within the broader research field of phototherapies, where dermatology is the most important discipline using and developing dosimetry in the UV, both with and without the use of photo-activatable molecules (e.g. photochemotherapy $[22,23])$. Intrinsically, UV dosimetry is not substantially different from visible light dosimetry, where the association of the "dose" concept to the incoming energy per unit area remains the golden standard up to now, unlike ionizing radiation dosimetry based on the absorbed and effective/equivalent dose concepts (Gy, Sv). This is why dosimetry in the UV-visible, and more recently near infrared, is so much strictly connected with efficacy/side-effect studies on the related biological effects (see next point). If on one side this seems to simplify the life of the medical physicist (irradiance calculation is probably easier that the calculation of an absorbed energy per unit mass) on the other it pushes towards the definition of more comprehensive ways to account for the physics of light-matter interaction to be related to the consequent biological response. From this point of view, treatment planning systems are already being developed for visible radiation, mainly in the anti-tumoral field, where most applications consider the insertion of optical fibers in precise positions into the organ to perform photodynamic therapy [24].

In the antimicrobial field, it seems to us that a lot of work could still be done from the dosimetry point of view, for example to understand if and how irradiation of a contaminated surface by UV light is intrinsically less effective that 3D bio-aerosol irradiation in terms of disinfecting capacity [25]. This is a tricky yet very important point, as it may drive the realization of innovative UV sources and light-delivery solutions.

Finally, from the point of view of UV-related biological effects, dermatology is again the most important application field for this subject $[19,26]$. At the same time, the advent of new sources and new ideas to bring light inside the human body $[27,28]$ opens the way to new scenarios where UV (and visible) light can be effectively used as a therapeutic agent against pathogens in additional medical disciplines. For all these reasons, antimicrobial applications are now increasingly studied to test their effectiveness and to better understand the principles at the basis of UV-induced photokilling in bacteria, viruses and fungi [29].

In this field the main working principle is certainly photo-chemical, where photoreactions are eventually followed by a biological response of the target leading to its death. In many studies, a phenomenological approach is used where observation of a certain dependence of the photokilling ratio on the irradiation parameters can be sufficient to derive indications on the best "irradiation protocol" in terms of wavelength, irradiance and irradiation time or timing. Nevertheless, the scientific community has been long developing research, both to increase the photokilling efficacy and to the better understand its molecular and biological bases $[26,30]$, gradually filling the gap between the in vitro and the in vivo. In this context, much work is dedicated to the optimization of the photodynamic principle, where photon absorption is eventually associated with the creation of cytotoxic reactive oxygen species [31,32]. All this is in the visible/near infrared range and, talking about antimicrobial applications, mainly in the antibacterial/ antifungal fields, while UV irradiation and viruses are still lacking comprehensive studies.

In conclusion, much more could be found and optimized in the UV range, especially in the UVC sub-range where new sources are being developed and above all new needs are strongly urging a response and a solution.

\section{Acknowledgements}

The authors would like to thank Prof. Piergiacomo Calzavara and Prof. Marina Venturini (University of Brescia, Italy) and Prof. Stefania Pallotta (University of Florence, Italy) for suggestions and revision of the manuscript.

\section{References}

[1] Shaman J, Kohn M. Absolute humidity modulates influenza survival, transmission, and seasonality. Proc Natl Acad Sci USA 2009;106:3243-8. https://doi.org/10. 1073/pnas.0806852106. 
[2] Modes of transmission of virus causing COVID-19: implications for IPC precaution recommendations n.d. https://www.who.int/news-room/commentaries/detail/ modes-of-transmission-of-virus-causing-covid-19-implications-for-ipc-precautionrecommendations (accessed May 19, 2020).

[3] Gazsó LG, Ponta CC. Radiation inactivation of bioterrorism agents. 1st ed. IOS Press; 2005.

[4] Buonanno M, Welch D, Shuryak I, Brenner DJ. Far-UVC light efficiently and safely inactivates airborne human coronaviruses. Res Sq 2020:1-21. https://doi.org/10. 21203/rs.3.rs-25728/v1.

[5] Buonanno M, Ponnaiya B, Welch D, Stanislauskas M, Randers-Pehrson G, Smilenov L, et al. Germicidal efficacy and mammalian skin safety of 222-nm UV Light. Radiat Res 2017;187:493-501. https://doi.org/10.1667/rr0010cc.1.

[6] Duguid JP. The size and the duration of air-carriage of respiratory droplets and droplet-nuclei. J Hyg (Lond) 1946;44:471-9. https://doi.org/10.1017/ S0022172400019288.

[7] Bourouiba L, Dehandschoewercker E, Bush JWM. Violent expiratory events: on coughing and sneezing. J Fluid Mech 2014;745:537-63. https://doi.org/10.1017/ jfm.2014.88.

[8] Erbil HY. Evaporation of pure liquid sessile and spherical suspended drops: a review. Adv Colloid Interface Sci 2012;170:67-86. https://doi.org/10.1016/j.cis. 2011.12.006.

[9] Liu L, Wei J, Li Y, Ooi A. Evaporation and dispersion of respiratory droplets from coughing. Indoor Air 2017;27:179-90. https://doi.org/10.1111/ina.12297.

[10] Tamerius JD, Shaman J, Alonso WJ, Bloom-Feshbach K, Uejio CK, Comrie A, et al. Environmental predictors of seasonal influenza epidemics across temperate and tropical climates. PLoS Pathog 2013;9. https://doi.org/10.1371/journal.ppat. 1003194.

[11] Bai Y, Levine M, Tumpey TM, Maines R. Tropism and infectivity of a seasonal A (H1N1) and a highly differentiated ferret nasal epithelial cell. Cultures 2019:1-16.

[12] Marr LC, Tang JW, Van Mullekom J, Lakdawala SS. Mechanistic insights into the effect of humidity on airborne influenza virus survival, transmission and incidence. J R Soc Interface 2019;16:20180298. https://doi.org/10.1098/rsif.2018.0298.

[13] Juzeniene A, Ma LW, Kwitniewski M, Polev GA, Lagunova Z, Dahlback A, et al. The seasonality of pandemic and non-pandemic influenzas: the roles of solar radiation and vitamin D. Int J Infect Dis 2010;14:e1099-105. https://doi.org/10.1016/j.ijid. 2010.09.002.

[14] Diffey BL. Human exposure to solar ultraviolet radiation. J Cosmet Dermatol 2002;1:124-30. https://doi.org/10.1046/j.1473-2165.2002.00060.x.

[15] Lytle CD, Sagripanti J-L. Predicted inactivation of viruses of relevance to biodefense by solar radiation. J Virol 2005;79:14244-52. https://doi.org/10.1128/jvi.79.22. 14244-14252.2005.

[16] Reed NG. The history of ultraviolet germicidal irradiation for air disinfection. Public Health Rep 2010;125:15-27. https://doi.org/10.1177/ 003335491012500105.

[17] Colella CM, Bogani P, Agati G, Fusi F. Genetic effects of UV-B: mutagenicity of 308 nm light in chinese hamster V79 cells. Photochem Photobiol 1986;43:437-42. https://doi.org/10.1111/j.1751-1097.1986.tb05626.x.

[18] Hamblin MR, Hasan T. Photodynamic therapy: a new antimicrobial approach to infectious disease? Photochem Photobiol Sci 2004. https://doi.org/10.1039/ b311900a.

[19] Gallagher RP, Lee TK. Adverse effects of ultraviolet radiation: a brief review. Prog
Biophys Mol Biol 2006;92:119-31. https://doi.org/10.1016/j.pbiomolbio.2006.02. 011.

[20] West S. Ocular ultraviolet b exposure and lens opacities: a review. J Epidemiol 1999;9:97-101.

[21] Kneissl M, Seong TY, Han J, Amano H. The emergence and prospects of deep-ultraviolet light-emitting diode technologies. Nat Photon 2019;13:233-44. https:// doi.org/10.1038/s41566-019-0359-9.

[22] Calzavara-Pinton P. Narrow band UVB (311 nm) phototherapy and PUVA photochemotherapy: a combination. J Am Acad Dermatol 1998;38:687-90. https://doi. org/10.1016/S0190-9622(98)70214-2.

[23] Hönigsmann H, Elmets CA, Krutmann J, editors. Dermatological Phototherapy and Photodiagnostic Methods. Berlin, Heidelberg: Springer Berlin Heidelberg; 2009. https://doi.org/10.1007/978-3-540-36693-5.

[24] Feather JW, Driver I, King PR, Lowdell C, Dixon B. Light delivery to tumour tissue through implanted optical fibres during photodynamic therapy. Lasers Med Sci 1990. https://doi.org/10.1007/BF02032590.

[25] Tseng CC, Li CS. Inactivation of virus-containing aerosols by ultraviolet germicidal irradiation. Aerosol Sci Technol 2005;39:1136-42. https://doi.org/10.1080/ 02786820500428575.

[26] Hamblin MR, Huang YY. Handbook of photomedicine. 2013. https://doi.org/10 1201/b15582.

[27] Lembo AJ, Ganz RA, Sheth S, Cave D, Kelly C, Levin P, et al. Treatment of Helicobacter pylori infection with intra-gastric violet light phototherapy: a pilot clinical trial. Lasers Surg Med 2009;41:337-44. https://doi.org/10.1002/lsm. 20770

[28] Tortora G, Orsini B, Pecile P, Menciassi A, Fusi F, Romano G. An ingestible capsule for the photodynamic therapy of helicobacter pylori infection. IEEE/ASME Trans Mechatron 2016;21. https://doi.org/10.1109/TMECH.2016.2536944.

[29] Dai T, Vrahas MS, Murray CK, Hamblin MR. Ultraviolet C irradiation: an alternative antimicrobial approach to localized infections? Expert Rev Anti Infect Ther 2012;10:185-95. https://doi.org/10.1586/eri.11.166.

[30] Wainwright M, Maisch T, Nonell S, Plaetzer K, Almeida A, Tegos GP, et al. Photoantimicrobials—are we afraid of the light? Lancet Infect Dis 2017;17:e49-55. https://doi.org/10.1016/S1473-3099(16)30268-7.

[31] Robertson CA, Evans DH, Abrahamse H. Photodynamic therapy (PDT): a short review on cellular mechanisms and cancer research applications for PDT. J Photochem Photobiol B Biol 2009. https://doi.org/10.1016/j.jphotobiol.2009.04. 001.

[32] Calzavara-Pinton P, Rossi MT, Sala R, Venturini M. Photodynamic antifungal chemotherapy. Photochem Photobiol 2012. https://doi.org/10.1111/j.1751-1097. 2012.01107.x.

Franco Fusi*, Giovanni Romano

Medical Physics Unit, Department of Experimental and Clinical Biomedical Sciences "Mario Serio" Viale G. Pieraccini 6, I-50139 Florence, University of Florence, Italy

E-mail addresses: franco.fusi@unifi.it (F. Fusi), giovanni.romano@unifi.it (G. Romano).

* Correspondig author. 